\title{
EL APRENDIZAJE DE LA RESPONSABILIDAD EN EL TRABAJO EN EQUIPO A TRAVÉS DE LA EVALUACIÓN FORMATIVA
}

Learning responsibility for teamwork through formative assessment

Financiado por la Convocatoria de Innovación 2016-2017 perteneciente a la Universidad de Zaragoza. Este estudio también se ha llevado a cabo dentro del proyecto de I+D+i: "La competencias docentes en la formación inicial del profesorado de educación física”. Convocatoria de noviembre de 2013 del Programa Estatal de Investigación, Desarrollo e Innovación Orientada a los Retos de la Sociedad, en el marco del Plan Estatal de Investigación Científica y Técnica y de Innovación 2013-2016. Referencia: EDU 2013-42024-R. Duración: 3 años (2014-2016).

\section{Sonia Asún Dieste (1) \\ Marta Rapún López (2)}

(1) Universidad de Zaragoza, España. Teléfono: +34 974238423. Correo electrónico: sonasun@unizar.es.

(2) Universidad de Zaragoza, España. Teléfono: +34 974238422 . Correo electrónico: mrapun@unizar.es

\section{Resumen}

Esta experiencia de innovación pretendió promover la responsabilidad de los estudiantes universitarios en el trabajo en equipo a través de un proceso de evaluación formativa. Se utilizaron rúbricas de coevaluación y autoevaluación, para medir y promover la responsabilidad en el trabajo en equipo. La muestra utilizada fue de 42 alumnos correspondientes a la Facultad de Ciencias de la Salud y del Deporte de Huesca. Se realizó un estudio pre-test, post-test. Se utilizó un cuestionario validado, en el que se especifican ítems relacionados con el trabajo en equipo (percepción de competencia de trabajo en equipo, de las habilidades interpersonales y de las intrapersonales) y se realizó un análisis cuantitativo descriptivo (frecuencias, porcentajes, media, mediana y desviación estándar). Los resultados mostraron la eficacia de esta experiencia para mejorar la responsabilidad y aumentar la percepción de competencia en el trabajo en equipo. 
Palabras clave: Trabajo en equipo; evaluación formativa; responsabilidad

\begin{abstract}
The aim of this innovation experience was to get responsibility of undergraduate students in teamwork through a formative assessment process. Student self and peer assessment rubrics was used to measure and promote responsibility in teamwork. The sample used was composed of 42 students from the Faculty of Health and Sport Sciences of Huesca. A pre-test post-test study was done. The validated questionnaire on competencies, in which different items related to team work were specified (teamwork, interpersonal and intrapersonal skills), was used, and a quantitative descriptive analysis (frequencies, percentages, mean, median and standard deviation) was done. The results showed the efficiency of the formative assessment to improve the responsibility and to increase the perception of teamwork competencies.
\end{abstract}

Keywords: Team work; formative assessment; responsibility

\title{
1. Introducción
}

El trabajo en equipo ha sido una de las competencias genéricas que los profesores universitarios han pretendido desarrollar en sus alumnos y alumnas con cierta asiduidad pero, con frecuencia, no se ha tenido en cuenta el proceso y el aprendizaje de las necesarias habilidades de los estudiantes para conseguir una óptima cooperación en el trabajo en equipo (Scallon, 2004). Sin embargo, ya hace algún tiempo que ciertos autores revelaban la importancia de la cooperación, la responsabilidad y las interrelaciones positivas de los estudiantes en sus trabajos en equipo, ofreciendo estrategias sobre cómo abordar estas cuestiones (Aylwin, 1994). Otros, debatían en torno a las calificaciones de los trabajos en equipo, pues éstas reflejaban resultados individuales más que del grupo (Howe, 2011); y otros, proponían la idoneidad de que se integraran valoraciones de profesores y estudiantes, mediante procesos de autoevaluación y co-evaluación (Chivite et al., 2015; Proulx, 2009). En este trabajo se pretendió recopilar todas estas reflexiones y elaborar una experiencia que consiguiese mejorar la capacidad de trabajar en equipo aumentando la responsabilidad de los estudiantes a través de la evaluación formativa con procesos de autoevaluación y coevaluación. 


\section{Contextualización}

La experiencia se realizó en el grado en Ciencias de la Actividad Física y del Deporte de la Universidad de Zaragoza, en la Facultad de Ciencias de la Salud y del Deporte (Campus Huesca).

En el curso 2016-2017, los 64 estudiantes de tercer curso de grado de la asignatura Diseño y evaluación de la enseñanza de la actividad física y del deporte, fueron informados sobre la posibilidad de participar en la experiencia. Finalmente, 42 aceptaron. Tenían edades comprendidas entre los 19 y los 29 años y el 80\% fueron hombres mientras que el $20 \%$ fueron mujeres.

\section{Diseño y desarrollo}

Esta experiencia pretendió promover la responsabilidad de los estudiantes universitarios en el trabajo en equipo a través de un proceso de evaluación formativa. El método fue la aplicación de una experiencia de innovación en un grupo de 42 estudiantes que debieron acometer el diseño, la aplicación y la defensa de un proyecto, trabajando en equipo. Se aplicaron acciones de evaluación formativa orientadas a la promoción y medida de la responsabilidad durante el proceso (fase inicial, intermedia y final), utilizando rúbricas elaboradas ad-hoc (Anexo 1), aunque adaptadas de otros trabajos existentes. Con ellas se obtuvo un perfil de responsabilidad de cada uno de los grupos tanto al principio como al final de la experiencia. Paralelamente se realizó un pre-test (principio de curso) y un post-test (final de curso) mediante la aplicación de un cuestionario de competencias validado por la Red nacional de evaluación formativa y compartida, en el que se especifican varios ítems relacionados con el trabajo en equipo. Se efectuó un análisis cuantitativo descriptivo (frecuencias, porcentajes, media, mediana y desviación típica).

\section{Evaluación}

Los resultados obtenidos sobre el nivel de responsabilidad de los equipos al final de la experiencia mejoran con respecto al inicio. Dos de los grupos (seminario 1 y 4 ) elevan sus porcentajes en los ítems relacionados con características de responsabilidad excelentes y admirables, mientras los otros dos grupos (seminario 2 y 3) también los elevan, pero en menor medida (Figura 1 y figura 2). 


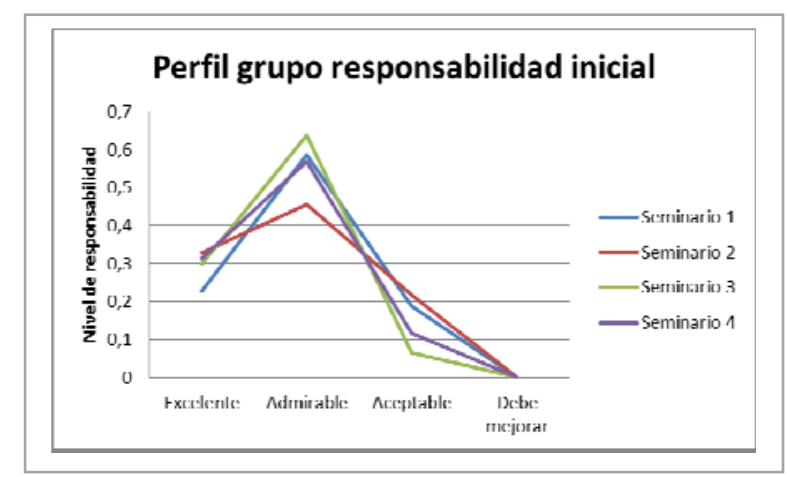

Figura 1.

Responsabilidad inicial.

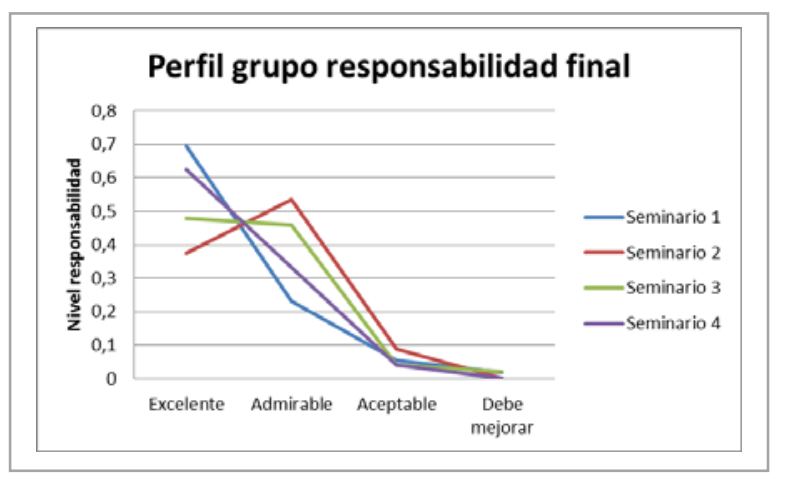

Figura 2.

Responsabilidad final.

Los resultados obtenidos sobre la competencia de los estudiantes en los ítems relacionados con la capacidad para trabajar en equipo, presentan una mejora entre los valores obtenidos en el pre-test $(\overline{\mathrm{x}}=3.17 ; \mathrm{Me}=3 ; \mathrm{DT}=16.61)$ y el post-test $(\overline{\mathrm{x}}=3.78$; $\mathrm{Me}=4 ; \mathrm{DT}=8.98)$.

Respecto a la competencia relacionada con las habilidades interpersonales (asertividad, empatía, respeto y escucha), la mejora fue algo más discreta entre el pretest $(\overline{\mathrm{x}}=3.13 ; \mathrm{Me}=3 ; \mathrm{DT}=23.22)$ y el post-test $(\overline{\mathrm{x}}=3.28 ; \mathrm{Me}=3 ; \mathrm{DT}=15.90)$, pasando de un porcentaje acumulado de $85 \%$ (situado entre mucha y bastante competencia), a un porcentaje de $90 \%$.

No se produjo incremento reseñable en la competencia relacionada con las habilidades intrapersonales (autoestima, motivación y confianza) entre el pre-test $(\overline{\mathrm{x}}=2.89 ; \mathrm{Me}=3 ; \mathrm{DT}=18.46)$ y el post-test $(\overline{\mathrm{x}}=2.90 ; \mathrm{Me}=3 ; \mathrm{DT}=13.60)$, donde, además, se bajó de un porcentaje acumulado de 82,6\% (entre mucha y bastante competencia) en el pre-test, a un porcentaje acumulado de 77,5\% en el post-test. Las emociones que se desatan ante los conflictos en grupo por diferencias personales, de motivación, etc...han 
estado muy presentes y, probablemente, el hecho de no ser competentes en la gestión de las emociones, puede haber provocado efectos contrarios a lo que se pretendía.

\section{Conclusiones y prospectivas}

Los resultados arrojan la eficacia de esta experiencia de evaluación formativa para mejorar la responsabilidad en el trabajo en equipo en este grupo de estudiantes, dado que el procedimiento consiguió elevar las cualidades positivas de los estudiantes, relacionadas con la responsabilidad y su competencia en el trabajo en equipo, así como su competencia interpersonal. Por el contrario, no logró tener incidencia positiva en la competencia intrapersonal, cuestión que debería ser abordada en futuros estudios y experiencias.

\section{Referencias}

Aylwin, U. (1994). Le travail en équipe : pourquoi et comment ?, Pédagogie collégiale, 7(3), 28-32.

Romero, M. R. Asún, S., \& Chivite, M. (2015). Coevaluación del diseño de unidades didácticas. En N. González, I. Salcines y García, E. (coords.), Tendencias actuales en evaluación formativa y compartida. El papel de las nuevas tecnologías. IX Congreso internacional de evaluación formativa y compartida en docencia infantil, primaria, secundaria y universitaria (pp. 1449-1466). Santander, España: Universidad Cantabria

Howe, R. (2011). Assessing Team work. College documentation bulletin, 7. Recuperado de http://regard.cdc.qc.ca/CDC/Pages/Front/Accueil/Accueil.aspx\#lisucc

Pastor del Pino, $\mathrm{M}^{\mathrm{a}}$ C. et al. (2017). Guía para la integración en la docencia de la competencia 2: TRABAJAR EN EQUIPO. Recuperado de http://repositorio.upct.es/bitstream/handle/10317/4100/gid2.pdf;jsessionid=57027 123D8722E071990F7F2612D1877?sequence=1

Proulx, J. (2009). Enseigner : réalité, réflexions et pratiques, Trois-Rivières : Cégep de Trois-Rivières.

Scallon, G. (2004). L'évaluation des apprentissages dans une approche par compétences. Saint-Laurent : Éditions du Renouveau Pédagogique. 


\section{ANEXO 1}

Nombre del alumno:

Cortar por aquí.

En los trabajos en equipo que hemos realizado normalmente este compañero o compañera

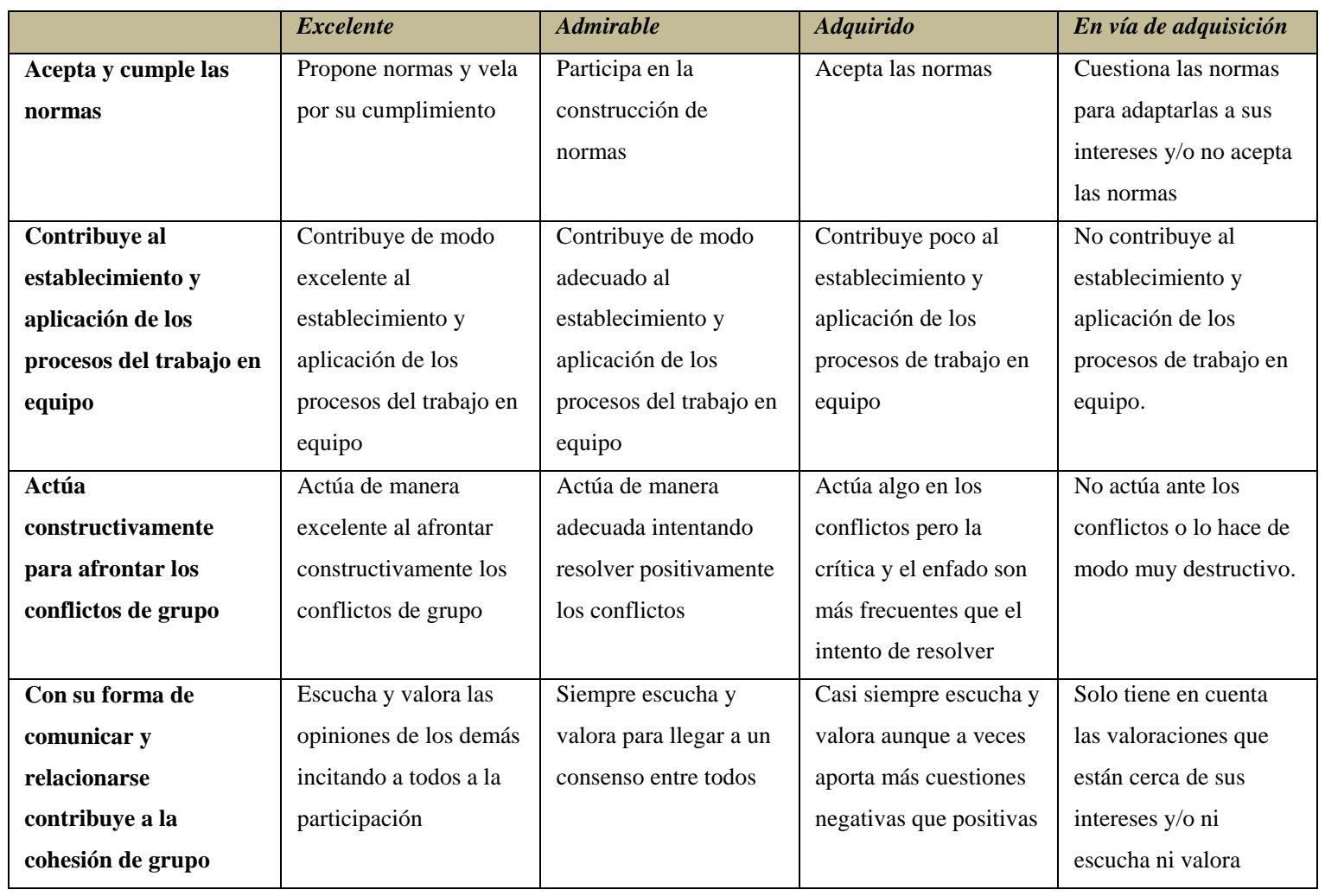

Adaptada de Pastor del Pino, $\mathrm{M}^{\mathrm{a}} \mathrm{C}$ y otros (2017). 